\title{
Percepções de interdisciplinaridade de professores de Ciências e Matemática: Um Exercício de Análise Textual Discursiva
}

\author{
Valderez Marina do Rosário Lima \\ \& Maurivan Güntzel Ramos
}

Resumo

O artigo apresenta resultados de investigação cujo tema central é a interdisciplinaridade. A pesquisa pretendeu responder à seguinte indagação: quais são as percepções de um grupo de professores que atuam na Educação Básica brasileira sobre interdisciplinaridade em sua relação com aprendizagem em Ciências e Matemática? Os sujeitos de pesquisa foram 40 professores ingressantes em um curso de pósgraduação em Educação em Ciências e Matemática oferecido por uma universidade situada ao sul do Brasil. A coleta dos dados ocorreu por meio de narrativas produzidas pelos participantes e, para tratamento das informações, utilizou-se o método de Análise Textual Discursiva. A análise originou três categorias, denominadas: Caracterizações de interdisciplinaridade; Interdisciplinaridade como ilusão de mudança no espaço pedagógico; Interdisciplinaridade como possibilidade real de mudança no espaço pedagógico. Os resultados do estudo mostram que os participantes da pesquisa percebem a interdisciplinaridade de modo pouco claro, superficial e genérico, associando o tema apenas à integração de disciplinas. Entretanto, o conceito está mais claro para um pequeno grupo de docentes que vinculou interdisciplinaridade ao uso de pesquisa em sala de aula.

\section{Palavras-chave:}

análise textual discursiva; interdisciplinaridade; ensino de Ciências e Matemática; percepções de professores; Educação Básica. 


\title{
Perceptions of interdisciplinarity of Science and Mathematic's teachers: an exercise of Discursive Textual Analysis
}

\begin{abstract}
The article presents the research's results which the central theme is the interdisciplinarity. The research aimed to answer the following question: What are the perceptions of a group of teachers, who work in the Brazilian Basic Education, about interdisciplinarity in its relationship with learning in Science and Mathematics? The research subjects were 40 incoming professors in a postgraduate course in Science and Mathematics Education offered by a university situated in the south of Brazil. Data collection was done through narratives produced by the participants and the information was analize using the Discursive Textual Analysis - ATD method. The analysis allowed the emergence of three categories: Characterization of interdisciplinarity; Interdisciplinarity as an illusion of change in the pedagogical space; Interdisciplinarity as a real possibility of change in the pedagogical space. The study's results have shown that the participants of the research perceive the interdisciplinarity in an unclear, superficial and generic way, associating the subject only to the integration of disciplines. However, the concept is clearer for a small group of teachers that linked interdisciplinarity to the use of classroom research.
\end{abstract}

Keywords: discursive textual analysis; interdisciplinarity; Science and Mathematics teaching; teachers' perceptions; Basic Education.

\section{Perceptions d'interdisciplinarité d'enseignats de Sciences e Mathématique : un exercice d'Analyse Textuelle Discursive (ATD)}

Résumé: Cet article présente les résultats d'une recherche centrée sur l'interdisciplinarité, répondant à la question: Quelles sont les perceptions d'un groupe d'enseignants travaillant à l'Education Basique Brésilienne à propos de l'interdisciplinarité en son rapport avec l'apprentissage en Sciences et Mathématique? Les sujets de la recherche sont 40 professeurs d'un Cours de Post-Graduation (40 professeurs suivant un cours de Post-Graduation) en Sciences et Mathématique offert par une université du sud du Brésil. Le prélèvement des données a été fait au moyen de récits produits par les participants et, pour le traitement des informations, on a utilisé la méthode d'Analyse Textuelle Discursive (ATD). L'analyse a permis l'émergence de trois catégories: Caractérisation d'Interdisciplinarité; Interdisciplinarité comme illusion de changement dans le domaine pédagogique; Interdisciplinarité comme possibilité réelle de changement dans le domaine pédagogique. Les résultats de cette étude montrent que les participants du sondage perçoivent l'interdisciplinarité de façon peu claire, superficielle et générique, n'associant le thème qu'à l'intégration de matières. Cependant, le concept est plus clair pour un petit groupe d'enseignants qui ont rapproché l'interdisciplinarité à l'usage de la recherche en salle de classe.

Mots-clés: analyse textuelle discursive ; interdisciplinarité; enseignement des Sciences et de Mathématique; perceptions des enseignants, Education basique.

\section{Percepciones de Profesores de Ciencias y Matemáticas acerca de la interdisciplinaridad: un ejercicio de la Análisis Textual Discursiva}

Resumen. El artículo presenta los resultados de la investigación cuyo tema central es interdisciplinaridad. La investigación buscó responder a la siguiente pregunta: ¿Cuáles son las percepciones de un grupo de profesores que trabajan en la Educación Básica brasileña, a respecto de la interdisciplinaridad y su relación con el aprendizaje en ciencias y matemáticas? Los participantes del estudio fueron 40 profesores ingresados recientemente en un curso de Postgrado en Educación en Ciencia y Matemáticas ofrecido por una universidad situada en el sur de Brasil. Los datos fueron colectados a través de narraciones producidas por los participantes y examinados por medio de la Análisis Textual Discursiva - ATD. El análisis hizo posible la emergencia de tres categorías. Caracterizaciones de la interdisciplinariedad, La interdisciplinaridad como una mudanza ficticia en el espacio educativo, La interdisciplinaridad se muestra como una posibilidad real de mudanza en el espacio educativo. Los resultados del estudio muestran que los participantes de la investigación perciben, la interdisciplinaridad, de forma poco clara, superficial e genérica, entendiendo el tema como, apenas, la integración de asignaturas. Entretanto, el concepto está más claro para un pequeño grupo de docentes que vinculó interdisciplinaridad al uso de la pesquisa en la sala de aula.

Palabras clave: análisis textual discursiva; interdisciplinaridad; enseñanza de ciencias y matemáticas; percepciones de los profesores; educación básica. 


\section{Introdução}

A interdisciplinaridade, tema central da investigação ora apresentada, encontra-se inserida em um cenário amplo, marcado pela complexidade que caracteriza a sociedade contemporânea e que repercute em várias esferas da vida humana e social. Tal complexidade alcança a educação e exige a reorganização dos modos de ensinar, tendo em vista a ampliação de oportunidades e de espaços para aprender, seja porque a produção de conhecimento ocorre celeremente, seja porque as tecnologias da informação e comunicação colocam o conhecimento à disposição de modo amplificado por meio de múltiplas ferramentas tecnológicas hoje disponíveis. O grande desafio da profissão docente hoje é buscar propostas que atendam às necessidades de formação do sujeito do século XXI, tanto no âmbito da formação científica quanto no que diz respeito aos atributos necessários para participar da vida em sociedade.

Neste cenário, a prática interdisciplinar surge como uma das soluções possíveis para a superação da fragmentação e descontextualização do conhecimento, bem como para a busca de soluções para problemas complexos (Morin, 2000). A interdisciplinaridade evidencia a necessidade de identificação das múltiplas perspectivas constituintes de um fenômeno/acontecimento, ensejando maior ligação, maior vínculo, entre os saberes das distintas áreas de conhecimento. Ao fazer isso, abre-se caminho para o encontro de soluções melhores, mais qualificadas, para os problemas que se impõem. Tal condição, entretanto, é observada com pouca frequência em ações disciplinares desenvolvidas nas salas de aula das escolas de Educação Básica, embora os documentos educacionais oficiais enfatizem a necessidade de um olhar interdisciplinar para o conhecimento, tendo em vista uma realidade social que se mostra cada vez mais complexa.

A pergunta é se os professores estão preparados para organizar e empreender um ensino com características integradoras e interdisciplinares. Assim, o presente estudo busca investigar essa problemática, tentando responder à seguinte questão:

- Quais são as percepções de um grupo de professores que atuam na Educação Básica brasileira sobre interdisciplinaridade em sua relação com aprendizagem em Ciências e Matemática?

A resposta a essa indagação foi construída por meio de análise de narrativas sobre o tema desenvolvidas por professores de Ciências e Matemática, as quais foram tratadas por meio da Análise Textual Discursiva - ATD (Moraes e Galiazzi, 2013). Esse procedimento de análise permitiu a emergência das percepções sobre a interdisciplinaridade desses docentes.

Este artigo encontra-se organizado em cinco seções, incluindo a Introdução, em que se justifica o estudo e se apresenta o problema da pesquisa. Na segunda seção, Interdisciplinaridade no contexto pedagógico, explicitam-se reflexões de teóricos so- 
bre a temática central da pesquisa. Na terceira seção, Desenho metodológico, explicase o desenho metodológico adotado, esclarecendo a abordagem e o tipo de pesquisa assumidos, definindo-se os sujeitos de pesquisa, a forma de coleta de dados e o método de análise utilizado. Na quarta seção, Resultados e discussão, apresentamse os resultados da análise empreendida, em um texto descritivo e interpretativo que evidencia as percepções sobre interdisciplinaridade dos docentes que fizeram parte do estudo. Na última seção, Conclusões principais, retoma-se o problema e, ao respondê-lo, apresentam-se algumas implicações da pesquisa, salientando aspectos a serem levados em conta quando se realizam atividades de educação continuada voltadas a professores de Ciências.

\section{Interdisciplinaridade no contexto pedagógico}

O extenso material disponível sobre interdisciplinaridade e o expressivo número de teóricos que se dedicam ao tema levam à constatação inicial de que interdisciplinaridade é um conceito trabalhado em muitas áreas de conhecimento. Em razão disso, é polissêmico, não havendo uma definição única estabelecida para o termo. Pombo (2013), ao discorrer sobre o assunto, chama atenção ao fato de a interdisciplinaridade estar na agenda das atividades de, no mínimo, quatro contextos atuais: o epistemológico, relativo ao trânsito de conhecimento entre disciplinas e pesquisadores; o midiático, no qual é fortemente presente; o empresarial e o tecnológico, ligados aos processos produtivos e de gestão; e, por fim, o pedagógico, em escolas e universidades, em que ocorre na interação de conhecimentos entre professores, alunos e disciplinas. Nele a interdisciplinaridade manifesta-se no currículo escolar por meio das metodologias adotadas. Para a autora, portanto, a apropriação por distintos ramos de atividade impede que a palavra tenha uma definição única, estável.

Quando se atenta ao contexto pedagógico, observa-se que a vinculação da interdisciplinaridade com a educação é também objeto de estudo e reflexão de muitos teóricos, dentre os quais citam-se Santomé (1998), Machado (1993), Koirala \& Bowman, 2003, Fazenda (2004), Frykholm \& Glasson, 2005, Maldaner (2014). Todos os teóricos aportam contribuições valiosas para qualificação de propostas pedagógicas integradoras. Entretanto, considera-se que o pensador francês Edgar Morin (2000), ao enunciar princípios para a educação do futuro, que para ele deve ser integral e interdisciplinar, acaba por apontar alguns caminhos possíveis para pensar projetos interdisciplinares a serem desenvolvidos na escola e na universidade. Para o autor, é essencial o desenvolvimento de ações que permitam reconhecer como conhecimento pertinente aquele que reflete sobre conhecimentos globais e conhecimentos parciais, situando, desse modo, as informações em um conjunto de maior complexidade e, segundo ele, para tal, "é preciso ensinar métodos que permitam estabelecer as relações 
mútuas e as influências recíprocas entre as partes e o todo num mundo complexo" (Morin, 2000, p. 14).

Nesse sentido, o trabalho pedagógico verdadeiramente interdisciplinar caracterizase pela integração que, segundo Sommerman (2012), é um atributo mais forte que interação e colaboração. É fazendo a distinção entre esses três termos que o autor organiza as definições de multi, pluri e interdisciplinar. Para ele, as ações integradas, prerrogativa da interdisciplinaridade, levam à síntese, à produção de algo novo e, por essa razão, geram avanço cognitivo. Tal condição não é alcançada na multidisciplinaridade, constituída por ações breves, sem continuidade, nem pela pluridisciplinaridade, que ocorre quando conceitos e métodos de uma disciplina são utilizados para auxiliar outra. Desse modo, é desejável que, ao assumirem a interdisciplinaridade como caminho possível para aprendizagem, os professores tenham claro que "o resultado da integração interdisciplinar [...] é algo totalmente novo. Vai além dos limites de qualquer disciplina [...]" (Sommerman, 2012, p. 571).

Com vistas a propiciar a integração, na perspectiva defendida por Sommerman (2012), o ensino basileiro estrutura-se em áreas (Linguagens, Ciências da Natureza, Ciências Humanas, Matemática), e as Diretrizes Curriculares Nacionais da Educação Básica referem que "a interdisciplinaridade pressupõe a transferência de métodos de uma disciplina para outra" (Brasil, 2013, p. 28), o que implica integração.

Assim, para ser efetiva, a presença de interdisciplinaridade no contexto pedagógico exigirá mais do que sua inclusão em planos; exigirá dos professores um entendimento que supere o delineamento de ações multi e pluridisciplinares.

\section{Desenho metodológico}

A pesquisa tem abordagem qualitativa, pois visa a compreender os modos de percepção dos professores em relação à interdisciplinaridade. Para isso, foi proposta aos sujeitos da investigação a seguinte questão: Narre alguma situação da sua experiência como professor(a) ou como aluno(a) que esteja relacionada à interdisciplinaridade no ensino de Ciências e Matemática.

Participaram do estudo 40 professores, sendo 14 do sexo masculino e 26 do sexo feminino. A idade variou de 22 a 50 anos, com média de 31 anos. Dos 40 sujeitos cujas narrativas foram analisadas, 18 são professores de Ciências (Física, Matemática e Química) e 22 de Matemática. Todos são ingressantes de um curso de pós-graduação em Educação em Ciências e Matemática. Os professores participantes da pesquisa são indicados por meio da letra "P", seguida de numeração sequencial, a fim de garantir o anonimato dos sujeitos que constituíram a amostra. Para apresentar as narrativas dos sujeitos, na análise desenvolvida no próxima seção, utiliza-se o texto grifado em itálico para diferenciarem-se das citações dos autores. 
Dentre os sujeitos da investigação, 30 professores atuam e têm experiência no magistério e 10 ainda não têm essa experiência. Quatro sujeitos têm formação em nível de pós-graduação stricto sensu (mestrado em Matemática, Ensino de Matemática e Ensino de Ciências) e 22 têm formação em nível lato sensu (como especialização em Metodologia em Ensino de Matemática, Gestão em Administração Pública, Docência em Ensino Superior, Docência em Saúde, Educação de Jovens e Adultos, Docência em Ensino Superior, Psicopedagogia Clínica e Institucional, Informática na Educação, Tecnologia em Educação a Distância, Educação Ambiental, dentre outros).

As respostas ao questionamento foram tratadas por meio da Análise Textual Discursiva - ATD (Moraes \& Galiazzi, 2013). A ATD é um método para analisar informações textuais de natureza qualitativa. Caracteriza-se por ser um processo auto-organizado, no qual as ideias do pesquisador vão se reconstruindo em relação ao corpus por ele estudado. Gradativamente, novos sentidos vão sendo concebidos sobre o objeto a ser conhecido. O rigor, que determina validade e confiabilidade dos resultados, concretizase pela intensa impregnação do investigador na realização da análise, em um processo de triangulação entre as fontes da investigação. A ATD é organizada em torno de quatro focos principais: unitarização, categorização, produção de metatextos e comunicação.

\section{Resultados e discussão}

As narrativas dos professores, ao serem submetidas à análise, inicialmente, são unitarizadas. A unitarização caracteriza-se pela desconstrução dos textos para identificar e isolar ideias com significado próprio. Desse processo de desmontagem originam-se as unidades de sentido, que são frases ou parágrafos expressando ideias individuais sobre o fenômeno estudado. Essa desmontagem dos textos, além de contribuir para a impregnação do pesquisador em relação ao corpus de análise, posibilita o processo de categorização. Neste estudo, a unitarização gerou 138 unidades de sentido relacionadas à questão de pesquisa.

A categorização é uma etapa em que as unidades de sentido são reunidas em categorias pela proximidade de significados. As primeiras categorias, denominadas iniciais, em geral são em grande número. Essas são reunidas em categorias intermediárias, as quais são novamente agrupadas em categorias finais. Essas categorias, portanto, emergem da ação interpretativa do pesquisador sobre corpus. Neste estudo, foram originadas 15 categorias iniciais, sete categorias intermediárias e três categorias finais.

A produção de metatextos consiste na elaboração de textos descritivos e, gradualmente, interpretativos para cada categoria em um processo rigoroso de análise. Para a produção dos metatextos é importante o diálogo com autores e teorias, as quais permitem iluminar o objeto de análise, conduzindo a uma nova organização do conjunto de ideias analisadas. As categorias intermediarias contribuem para estruturar as ideias 
desenvolvidas no metatexto, sem a necessidade de mencioná-las na forma de subcategorias (subtítulos). Ainda, na construção de metatextos, algumas das unidades de sentido são citadas para fortalecer os argumentos desenvolvidos.

A comunicação é a etapa da análise na qual são explicitados os argumentos construídos ao longo do processo de análise por meio de artigos, os quais passam a ser validados em novos contextos.

As narrativas dos professores, ao serem submetidas à análise, originaram três categorias. Uma delas reuniu os entendimentos sobre o termo interdisciplinaridade e se intitulou Caracterizações da interdisciplinaridade. Em suas reflexões, focalizando a interdisciplinaridade no âmbito pedagógico, os professores expuseram ainda suas crenças sobre a relação entre interdisciplinaridade e aprendizagem. Assim, as duas outras categorias foram elaboradas com base nas referidas percepções. A segunda categoria de análise, denominada Interdisciplinaridade como ilusão de mudança no espaço pedagógico, trata das declarações de professores que percebem a interdisciplinaridade como receita, como método quase infalível para a realização de aprendizagem pelos alunos. A terceira categoria, denominada Interdisciplinaridade como possibilidade real de mudança no espaço pedagógico, reúne as ideias daqueles docentes para quem ações interdisciplinares apresentam grande potencial para a ocorrência de aprendizagem. Apresentam-se, a seguir, os metatextos com as descrições e interpretações realizadas em cada uma das categorias emergentes do processo de análise.

\subsection{Categoria 1 - Caracterizações de interdisciplinaridade}

As declarações dos entrevistados dadas como respostas à questão proposta foram organizadas em torno de duas ideias principais: formulação de tentativas de definições para o termo; reflexões sobre as finalidades da interdisciplinaridade, associadas a configurações que ela pode assumir para concretizar-se. Em relação à primeira ideia, encontram-se professores que referiram ser a interdisciplinaridade:

"[...] uma integração entre disciplinas" (P1);

"[...] a integração das ciências" (P8);

ou "Inter significa a utilização de procedimentos de uma área em outra área do conhecimento" (P11);

"[...] uma interação pode ir da simples comunicação de ideias à integração mútua dos conceitos diretores da epistemologia, da terminologia, da metodologia, dos procedimentos, dos dados e da organização referente ao ensino e à pesquisa" (P15); 
ou também "[...] o objeto a ser estudado é comum a dois ou mais campos disciplinares inter-relacionados" (P18);

é um processo que "[...] se apoia numa visão de mundo que considera que as diversas partes da realidade interagem entre si, não sendo possível compreender um sistema complexo com base na compreensão de suas partes isoladas" (P35).

As percepções de todos os entrevistados estão representadas nas citações mencionadas. Embora as ideias apontadas sejam semelhantes, apresentam nuances, indicando o entendimento de que interdisciplinaridade pode ser integração de disciplinas (P1, P18), de área de conhecimento (P8), de métodos (P11), de partes de um sistema (P35) e de conceitos (P15).

Em relação à segunda ideia, incluíram-se declarações como: "interdisciplinaridade é um assunto abordado pelo maior número de componentes curriculares, dentro da dinâmica do ano ou série que se esteja trabalhando"(P7); "[...] por exemplo, o estudo do comportamento das partículas na Física sendo utilizado na Química, utilização de um método em diferentes áreas do conhecimento"(P11); "[...] integrar diversas disciplinas para o aprendizado de um determinado conteúdo, exemplo, estudar o sistema respiratório com conceitos da Biologia, Química, Matemática, entre outras. Interdisciplinaridade seria usar das diversas disciplinas para estudar um tema" (P39).

O olhar sobre esse material permite que sejam feitas algumas considerações. Em primeiro lugar, os docentes, em vez de enunciarem definições para interdisciplinaridade, apresentaram elementos concretos de modo a posibilitar ao leitor o reconhecimento desse conceito (P7, P11 e P39). Em segundo lugar, entre os textos de professores que seguiram o caminho da teorização, apresentando uma definição, depreende-se que nenhuma delas pode ser considerada completa, embora cada uma explicite um, ou mais, elementos fundamentais para a definição do termo, principalmente quando são comparadas à definição de Sommerman (2012, p. 491) em estudo recente:

[...] interação prolongada e coordenada entre disciplinas acadêmicas para a compreensão de determinados temas ou a resolução de determinado problema que não pode ser tratado adequadamente por abordagens monodisciplinares; essa interação levando muitas vezes à integração dos diferentes discursos das disciplinas mediante a criação de uma linguagem ou de um quadro conceitual comum, chegando a formular uma metodologia comum, transcendendo ou na interface, das epistemologias de diferentes disciplinas e gerando um conhecimento novo.

A falta de clareza dos professores sobre o conceito de interdisciplinaridade também foi relatado por Mozena e Ostermann (2014). Nesse material, a recorrência de menções relacionando interdisciplinaridade e aprendizagem chamou a atenção dos 
pesquisadores e, por ser a aprendizagem dimensão primordial do processo educativo, decidiu-se pela continuidade da análise sob tal perspectiva.

\subsection{Categoria 2 - Interdisciplinaridade como ilusão de mudança no espaço pedagógico}

Ao relatarem experiências consideradas interdisciplinares, alguns docentes citam "ações em que dois professores entram juntos em sala de aula e cada um expõe diferentes perspectivas de um mesmo conceito com o intuito de melhor explicar o fenômeno" (P9). Para o Professor 8, desse modo o ensino assume uma "configuração que possibilita a interação e facilita o entendimento e a aprendizagem do aluno". Encontram-se nesse grupo, também, professores que mencionam que "interdisciplinaridade é juntar disciplinas diferentes para desenvolver um mesmo conceito ou fato, o que facilita a compreensão, a contextualização e, consequentemente, a aprendizagem dos alunos" (P14) e, ainda, narrativas como o de P20 ao referir como experiência interdisciplinar a atividade "sobre tecnologia na qual trabalhamos o programa Excel na construção de planilhas e pequenas noções de Matemática".

As manifestações anteriormente apresentadas expressam diferentes ideias de parte dos sujeitos de pesquisa. Observa-se, primeiramente, a tendência dos docentes a referirem-se à interdisciplinaridade como uma proposta de trabalho ou, até, como uma estratégia de ensino capaz de por si só favorecer o processo de aprender (P8 e P9). Em segundo lugar, há indícios de que, para os professores em questão, há uma relação direta entre tratar um tema, sob distintas óticas, e construir conhecimento sobre ele, ou seja, o grupo confunde abordar um conteúdo de modo diferenciado com aprender esse conteúdo, confunde ensino com aprendizagem (P14 e P20). Para eles, o fato de reunir dois professores, ou mesmo de um só docente discorrer sobre aspectos de um fenômeno que pertençam a diferentes áreas de conhecimento torna-se, no caso, ato suficiente a fim de produzir aprendizagem para aqueles que ouvem as explicações. Não está presente, nas declarações dos docentes, argumentação consistente sobre como - e por que - os estudantes aprenderiam melhor quando os conceitos são trabalhados, pelo professor, apresentando-o sob diferentes óticas, nas situações apresentadas. Além disso, a ausência de reflexão mais profunda parece estar associada à falta de clareza sobre interdisciplinaridade e também sobre os modos como os sujeitos aprendem.

Ressalta-se, também, a forte centralidade atribuída ao professor em ações consideradas interdisciplinares, como demonstram as afirmações a seguir: "O educador deve saber interpretar um fenômeno da natureza de forma holística, utilizando o conhecimento das disciplinas e inter-relacioná-los para poder ampliar sua problemática" (P16); "uma atividade ou um projeto que a maioria dos professores possa trabalhar os assuntos por meio de atividades que contemplem seus conteúdos” (P28 ); e, ainda, 
P29 que cita como modelo de interdisciplinaridade um "experimento, envolvendo Ciências e Matemática. No experimento, introduzimos juntamente aos alunos noções básicas sobre medidas de volume e $\mathrm{pH}$, relacionamos a ciência Química com a Matemática através do estudo da composição de água em alimentos". Nos exemplos apresentados, constata-se uma visão ingênua de interdisciplinaridade ao atribuir ao professor o papel central no processo de integração dos conceitos, pois, na perspectiva dos entrevistados, é o professor que relaciona as áreas de conhecimento, que busca pontos em comum, que dá sentido às informações. Entretanto, além de interdisciplinaridade estar relacionada à ideia de articulações complexas para que determinado fenômeno possa ser compreendido de forma ampla é, também, um conceito relacionado ao desenvolvimento de atitudes como curiosidade e abertura de espírito, conforme assevera Pombo (2005, p.13) em suas reflexões:

Interdisciplinaridade se deixa pensar, senão apenas na sua faceta cognitiva - sensibilidade à complexidade, capacidade de procurar mecanismos comuns, atenção a estruturas profundas que possam articular o que aparentemente não é articulável - mas também em termos de atitude: curiosidade, abertura de espírito, gosto pela cooperação, pelo trabalho em comum.

Na perspectiva pedagógica, o desenvolvimento de atitudes, e mesmo a capacidade de pensar a complexidade do objeto cognoscível, são transformações desejáveis de ocorrer no sujeito aprendente, isto é, no estudante. A responsabilidade do docente no processo não é a de explicar todas as possíveis leituras de um conceito/fenômeno, mas é a de criar situações de ensino que contribuam para que os estudantes construam atitudes de pensar em rede de significados, acolhendo múltiplas perspectivas que podem contribuir para qualificar seu entendimento sobre o conteúdo estudado. São exercícios de tal natureza que podem encaminhar a construção do que Morin (2000) denomina reforma do pensamento, condição necessária para promover articulação e organização de conhecimentos sobre o mundo contemporâneo. Entretanto, como afirma o filósofo e sociólogo francês, "esta reforma é paradigmática e, não, pragmática: é a questão fundamental da educação, já que se refere a nossa aptidão de organizar o conhecimento" (Morin, 2000, p. 35).

Os professores cujas narrativas foram até aqui apresentados percebem haver relação entre interdisciplinaridade e aprendizagem, mas não possuem clareza sobre aspectos importantes que determinam tal vinculação. Porém, no conjunto dos professores que fizeram parte do estudo, há outros que, ao expressarem suas ideias, apontam elementos para justificar suas posições sobre a conexão existente entre aprendizagem e interdisciplinaridade, conforme apresentado na discussão da categoria a seguir. 


\subsection{Categoria 3 - Interdisciplinaridade como possibilidade real de mudança no espaço pedagógico}

Os docentes que se situam no grupo cujas percepções são apresentadas nesta categoria discutem interdisciplinaridade na lógica da aprendizagem, e não na do ensino. Pelas experiências interdisciplinares por eles relatadas, ações dessa natureza criam condições para que o ato de aprender se efetive, como pode ser visto na declaração de P13, para quem interdisciplinaridade é "possibilitar a formação de educandos com uma visão holística de saberes, fazendo conexões, unindo teoria e prática de forma crítica e reflexiva"; para P32, que a percebe como "modo de promover a educação de forma que o aluno aprenda a utilizar os conhecimentos, identificando as várias faces do conceito"; e para P38 que, ao defender a superação da compartimentalização das áreas de saber, comenta que assim o "[...] aluno cria conexões e percebe que o conhecimento não deve ser compartimentado e sim integrado".

$\mathrm{Na}$ base das afirmações antes mencionadas, situa-se a percepção de que experiências assim delineadas permitem ao sujeito aprendente desenvolver um modo particular de conduzir o pensamento, ou seja, um modo orientado para a visão global dos acontecimentos e pela busca de conexões entre partes que constituem o fenômeno/ conceito, promovendo, desse modo, a aprendizagem de uma atitude, de uma forma de ver o mundo (P13, P32, P38).

Os professores ainda explicitam aspectos associados à atividade interdisciplinar, como o trabalho efetivamente integrado, e a concretização dessas ações por meio de feiras e mostras escolares. Como exemplo do primeiro ponto, cita-se a experiência relatada por P8, que na condição de participante de um minicurso vivenciou uma experiência "divina", conforme suas palavras. Conta ela que "as professoras, uma graduada em Química e a outra em Biologia, demonstraram como é possível trabalhar em sala de aula de forma interdisciplinar. Cada uma delas contribuiu com o conhecimento de sua área, sendo complementado pelo conhecimento da outra, sem superposição, apenas se completando". O sujeito P8 finaliza mencionando ter compreendido que "é possível essa configuração, que possibilita uma interação entre os conhecimentos, facilitando o entendimento e a aprendizagem dos alunos". No exemplo trazido por P8, está presente um dos pilares da interdisciplinaridade que é a integração coordenada (Sommerman, 2012).

A realização de eventos como feiras e mostras foi citada por alguns dos sujeitos que fizeram parte do estudo e, dentre eles, destaca-se o depoimento de P3 que refere a Feira de Ciências e de Matemática, atividade desenvolvida com o intuito de que os alunos estudassem o Reino Vegetal. No período preparatório, os alunos estudaram diversos conteúdos constituintes da temática e elaboraram "objetos ou alimentos com os vegetais para vendê-los aos visitantes da feira, geralmente, pais e familiares. Dentre a produção para a venda estavam: copos/vasos com mudinhas de plantas, bolinhos 
de espinafre, saladas e chás de ervas medicinais, por exemplo". Prossegue ele afirmando que: "[...] esta experiência interdisciplinar possibilitou o uso de conhecimentos de Ciências, na elaboração das bancas, e explicação sobre o Reino Vegetal; de Matemática, nas anotações financeiras, o uso das quatro operações básicas dependendo da situação da venda dos objetos ou alimentos".

As feiras de Ciências têm entre seus objetivos a apresentação de trabalhos que sejam de natureza investigativa, isto é, a pesquisa é o eixo estruturador das atividades desenvolvidas. É possível dizer, então, que alguns professores têm a percepção de que a pesquisa no âmbito escolar é um modo de desenvolver atividades interdisciplinares. Autores como Demo (2007), Moraes (2008), Ramos (2008) e Maldaner (2014), dentre tantos outros, defendem o uso da pesquisa em ambiente escolar e salientam suas qualidades para organizar situações de aprendizagem estruturadas de forma a colocar o estudante na centralidade do processo de aprender. Ora em parceria com colegas, ora de modo individual, o estudante lança mão de conceitos e informações provenientes de diversas áreas do conhecimento e elabora sínteses provisórias, cada vez mais complexas, que expressam a ampliação de seu conhecimento inicial sobre os conceitos trabalhados. A reflexão sobre pesquisa escolar aqui apresentada encontra ressonância nas ideias de Paviani (2008) ao referir que os elementos que caracterizam um ato interdisciplinar não são estabelecidos na exterioridade, mas que "os verdadeiros critérios são os epistemológico-pedagógicos, isto é, os que surgem do interior do processo de ensino aprendizagem e das necessidades sociais e morais" (p. 113). O uso da pesquisa parece ser um caminho fecundo para a realização de ações interdisciplinares amparadas nos verdadeiros critérios mencionados pelo autor. Assim, a pesquisa como ato interdisciplinar cria condições para o que os estudantes aperfeiçoem o pensamento complexo, pensamento esse que privilegia as relações intrínsecas entre múltiplos prismas que compõem um conceito/fenômeno importante de ser estudado.

Para finalizar a análise, retoma-se a proposição teórica de Pombo (2013) de que, ao se falar de interdisciplinaridade, refere-se a um continuum com níveis que possuem diferentes graus de interação. Entende-se que entre os professores, sujeitos da pesquisa em tela, encontram-se aqueles para quem basta haver um grau mínimo de coordenação para que a atividade seja definida como interdisciplinar, o que, segundo a autora, representam integrações multi/pluridisciplinares. Mas há outro grupo de docentes para os quais uma ação interdisciplinar só pode ser assim considerada se houver convergência de pontos de vista, alinhando-se à proposição postulada por Pombo. Assume-se, nesta investigação o posicionamento de que, embora os docentes não estejam totalmente apropriados dos princípios que fundamentam o ato interdisciplinar 
na perspectiva pedagógica, os professores dão visibilidade a ações com valor interdisciplinar e são capazes de associá-las à ocorrência de aprendizagem pelos estudantes que as experenciam. Salienta-se, também, que a visão interdisciplinar, sendo essencial na formação do sujeito contemporâneo, precisa ser mais estudada e mais bem compreendida por professores e futuros professores.

\section{Conclusões}

Com a intenção de investigar as percepções sobre interdisciplinaridade, 40 professores de Ciências e Matemática foram questionados no sentido de apresentarem narrativas de situações concretas vivenciadas, nas quais estava presente a interdisciplinaridade. Esses docentes tinham em comum a condição de serem ingressantes em um programa de pós-graduação nos cursos de mestrado ou doutorado em Educação em Ciências e Matemática.

Considerando o foco central da investigação, destaca-se a falta de clareza em relação a uma definição consistente de interdisciplinaridade, pois os professores apresentavam apenas alguns indícios sobre esse conceito, os quais estavam associados somente à integração entre disciplinas, áreas de conhecimento ou conceitos. Além disso, as definições apresentadas são parciais em relação às definições de autores referências sobre o assunto.

Por outro lado, há narrativas que mostram possibilidades reais de melhoria da aprendizagem por meio de ações interdisciplinares, principalmente quando a pesquisa em sala de aula é mencionada e exemplificada, como o caso de participação em atividades que desencadeiam nas feiras ou mostras de Ciências e Matemática.

Desse modo, a investigação mostra haver necessidade de ações educativas nos processos de formação de professores, inicial e continuada, que contemplem o experienciar de projetos interdisciplinares. Para a ocorrência de avanços em termos de clareza conceitual, tanto no âmbito teórico quanto na perspectiva da prática, é essencial o estudo e a discussão entre professores de diferentes áreas não só sobre interdisciplinaridade, mas também sobre as relações possíveis de serem efetivadas em contextos marcados pela disciplinaridade, como é, na maioria das vezes, a organização curricular das escolas. É o entendimento das dimensões epistemológicas e metodológicas do conceito que podem evitar o uso vazio e superficial do termo, conforme se constata na percepção de alguns docentes.

Por fim, o estudo apresentado pode contribuir para importantes reflexões dos professores sobre as relações entre o conceito interdisciplinaridade e aprendizagem nas áreas de Ciências e Matemática. 


\section{Referências Bibliográficas}

Brasil. Ministério da Educação. (2013). Diretrizes Curriculares Nacionais para a Educação Básica. Brasilia: MEC, SEB, DICEI, 6-79.

Demo, P. (2007). Educar pela pesquisa. Campinas: Autores Associados.

Fazenda, I. C. A. (Org.). (2004). Novos enfoques da pesquisa educacional. São Paulo: Cortez.

Frykholm, J. \& Glasson, G. (2005). Connecting science and mathematics instruction: Pedagogical context knowledge for teachers. School Science and Mathematics, 105 (3), 127-141.

Furner, J. M. \& David D. K. (2007). The Mathematics and Science Integration Argument: A Stand for Teacher Education. Eurasia Journal of Mathematics, Science \& Technology Education, 3(3), 185189.

Koirala, H. P. \& Bowman, J. K. (2003). Preparing middle level preservice teachers to integrate mathematics and science: Problems and possibilities. School Science and Mathematics, 145(10), 145-154.

Machado, N.J. (1993). Interdisciplinaridade e Matemática. Pro-Posições, (14)1, 185-206.

Maldaner, O. A. (2014). Formação de Professores para um Contexto de Referência Conhecido. In Nery, B. \& Maldaner, O. A. Formação de Professores: Compreensões em Novos Programas e Ações. (pp.15-41) ljuí: Unijuí.

Moraes, R. (2008). Cotidiano no ensino de Química: superações necessárias. In Galiazzi, M.C.; Auth, M.; Moraes, R.: Mancuso, R. Aprender em rede na educação em Ciências. (pp.15-34). Porto Alegre: Edipucrs.

Moraes, R.; Galiazzi, M. do C. (2013). Análise Textual Discursiva. ljuí: Unijuí.

Morin, E. (2000). A cabeça bem-feita. Repensar a reforma; reformar o pensamento. Rio de Janeiro: Bertrand Brasil.

Mozena, E.R.; Ostermann, F. (2014). Uma revisão bibliográfica sobre a interdisciplinaridade no ensino das Ciências da Natureza. Revista Ensaio, (16) 2, 185-206.

Paviani, J. (2008). Interdisciplinaridade: conceitos e distinções. Caxias do Sul: Educs.

Pombo, O. (2005) Interdisciplinaridade e integração de saberes. Liinc em Revista, 1, 3 -15 . Disponível em http://www.ibict.br/linc, consultado em 12.12.2016.

Pombo, O. (2013). Epistemologia de la interdisciplinaridade. La construcción de um nuevo modelo de comprensión. Intedisciplina I, 1(1), 21-50.

Ramos, M.G. (2008). A importância da problematização no conhecer e no saber em Ciências. In Galiazzi, M.C.; Auth, M.; Moraes, R.: Mancuso, R. Aprender em rede na educação em Ciências. (pp.57-76). Porto Alegre: Edipucrs.

Sommerman, A. (2012). A interdisciplinaridade e a transdisciplinaridade como novas formas de conhecimento para a articulação de saberes no contexto da ciência e do conhecimento em geral: contribuição para os campos da Educação, da Saúde e do Meio Ambiente. Tese de Doutorado em Difusão do Conhecimento, Faculdade de Educação, Universidade Federal da Bahia, Salvador.

Santomé, J. T. (1998). Globalização e interdisciplinaridade. Porto Alegre: Artmed. 
Valderez Marina do Rosário Lima Programa de Pós-Graduação em Educação, Faculdade de Educação e Programa de Pós-Graduação em Educação em Ciências e Matemática

- Faculdade de Física, PUCRS - Pontifícia Universidade Católica do Rio

Grande do Sul

Valderez.lima@pucrs.br

Maurivan Güntzel Ramos

Faculdade de Química e Programa de Pós-Graduação em Educação em Ciências e Matemática - Faculdade de Física, PUCRS - Pontifícia

Universidade Católica do Rio Grande do Sul

Correspondência

Valderez Marina do Rosário Lima

Programa de Pós-Graduação em Ciências e Matemática - Faculdade de

Física, PUCRS

Av. Ipiranga, 6681, prédio 10, sala 208, Porto Alegre, RS, Brasil, CEP

90610-900

Data de submissão: Julho 2016

Data de avaliação: Outubro 2016

Data de publicação: Julho 2017 